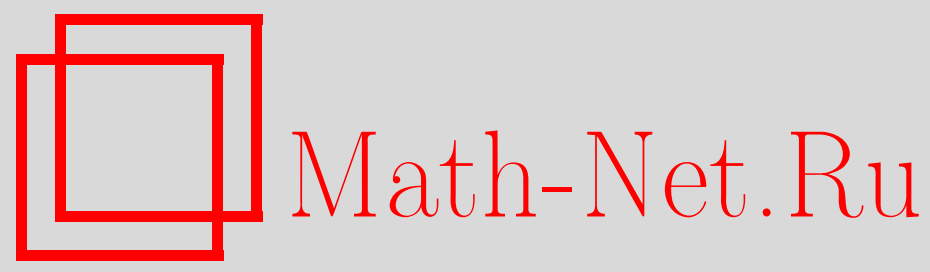

Р. Б. Салимов, Н. К. Туктамышов, Решение задачи Гильберта для кольца в особом случае и его применение к одной задаче взрыва, Матем. заметки, 1999, том 66, выпуск 1, 135-144

DOI: https://doi.org/10.4213/mzm1148

Использование Общероссийского математического портала Math-Net.Ru подразумевает, что вы прочитали и согласны с пользовательским соглашением http://www.mathnet.ru/rus/agreement

Параметры загрузки:

IP : 34.239 .49 .27

26 апреля 2023 г., 10:32:10

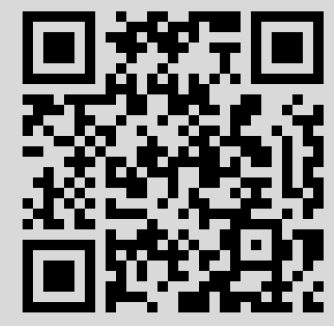




\title{
РЕШЕНИЕ ЗАДАЧИ ГИЛЬБЕРТА ДЛЯ КОЛЬЦА В ОСОБОМ СЛУЧАЕ И ЕГО ПРИМЕНЕНИЕ К ОДНОЙ ЗАДАЧЕ ВЗРЫВА
}

\author{
Р. Б. Салимов, Н. К. Туктамышов
}

В статье приведен метод решения краевой задачи Гильберта с разрывными коэффициентами для аналитической и однозначной в кольце функции, когда решение может иметь степенные особенности в конечном числе точек на границе кольца. В качестве применения полученных результатов рассмотрена задача о взрьве двух шшуровых зарядов в однородной среде, когда в ней имеется круговой цилиндр, обтекаемый возникаюшим при взрыве течением.

Библиограффия: 10 названий.

Пусть в плоскости комплексного переменного $z=x+i y$ дано кольцо $D: q<|z|<1$ и $L=L_{0}+L_{1}-$ граница области $D$, где $L_{0}$ есть окружность $|z|=q$. За положительное направление обхода примем такое, при котором область $D$ остается слева. Требуется определить функцию $F(z)=u(z)+i v(z)$, аналитическую и однозначную в $D$, кроме конечного числа точек $t_{j, k_{j}}, k_{j}=1, \ldots, p_{j}, j=0,1$, вблизи которых $F(z)$ может иметь степенные особенности порядка не вьше $m_{j, k_{j}}+\lambda_{j, k_{j}}$, где $m_{j, k_{j}}$ - целоенеотрицательное число, $0 \leqslant \lambda_{j, k_{j}}<1, k_{j}=1, \ldots, p_{j}$, по краевому условию

$$
\operatorname{Re}\{(a(t)+i b(t)) F(t)\}=\frac{c(t)}{\prod_{j=0,1} \prod_{k_{j}=1}^{p_{j}}\left|t-t_{j, k_{j}}\right|^{m_{j, k_{j}}+\mu_{j, k_{j}}}},
$$

где $0 \leqslant \mu_{j, k_{j}}<1, a(t), b(t), c(t)$ - заданные на $L$ действительные функции, имеющие разрьвы первого рода в точках $t_{j, k_{j}}, k_{j}=1, \ldots, p_{j}, j=0,1$, и удовлетворяющие условию Гёльдера на каждой из дуг $t_{j, k_{j}}, t_{j, k_{j}+1}, k_{j}=0, \ldots, p_{j}, j=0,1$, включая конщы, причем $t_{j, 0}=t_{j, p_{j}+1}=q^{j}, j=0,1$. Считаем, что точки $t=1$ и $t=q$ не являются точками разрыва коэффициентов условия (1). При этом будем считать, что при положительном обходе контура $L$ за точкой $t_{j, k_{j}}$ следует точка $t_{j, k_{j}+1}, k_{j}=0, \ldots, p_{j}, j=0,1$. Предположим, что $a^{2}(t)+b^{2}(t) \neq 0$ всюду на $L$.

Особый случай задачи Гильберта для круга рассмотрен в статье [1]. Как известно, $\Phi$. Д. Гахов [2, с. 264-289] дал эффективное решение краевой задачи Гильберта для односвязной области, основанное на отыскании регуляризующего множителя. Используя метод построения однозначного регуляризующего множителя, Э. И. Зверович [3] получил решение этой задачи для многосвязной области.

Вовсех упомянутых работах коэффициенты краевого условия предполагаются непрерьвньми. В настоящей статье метод, разработанньй Зверовичем, с некоторыми изменениями обобщается на случай разрывных коэффициентов, когда область имеет форму кольца. 
Отметим, что большое число задач из различных областей гидродинамики (в частности, теории безотрьвных, струйных, кавитационных течений невязкой жидкости [4], [5], теории фильтрации [6], взрьва [7] и т.д.) в математической постановке приводятся к нахождению аналитической функции в канонических областях с краевыми условиями вида (1). Вместе с тем решение многих из них имеет довольно громоздкий вид и включает в себя большое число различных специальных функций. В данной работе приводится достаточно простой метод решения задачи (1), удобньй и с точки зрения приложений. В частности, в качестве иллюстрации приведено решение одной задачи взрьва.

Решение задачи будем искать в виде

$$
F(z)=\frac{F_{1}(z)}{\prod_{k_{0}=1}^{p_{0}}\left(z-t_{0, k_{0}}\right)^{m_{0, k_{0}}} \prod_{k_{1}=1}^{p_{1}}\left(\frac{z-t_{1, k_{1}}}{z}\right)^{m_{1, k_{1}}}},
$$

где $F_{1}(z)$ - аналитическая и однозначная в области $D$ функция, имеющая, быть может, лишь интегрируемые особенности в точках $t_{j, k_{j}}, k_{j}=1, \ldots, p_{j}, j=0,1$.

Обозначим через $\theta_{0, k_{0}}(t)=\arg \left(t-t_{0, k_{0}}\right)$ граничное значение непрерьвной в $D$ ветви $\arg \left(z-t_{0, k_{0}}\right)$, которая при $t=\exp (i \gamma)$ принимает значение $\theta_{0, k_{0}}=3 \pi / 2+$ $\left(\gamma+\gamma_{0, k_{0}}\right) / 2$ при $0 \leqslant \gamma<\gamma_{0, k_{0}}, \theta_{0, k_{0}}=\pi / 2+\left(\gamma+\gamma_{0, k_{0}}\right) / 2$ при $\gamma_{0, k_{0}}<\gamma \leqslant 2 \pi\left(\gamma_{0, k_{0}}=\right.$ $\left.\arg t_{0, k_{0}}\right), \theta_{1, k_{1}}(t)=\arg \left(\left(t-t_{1, k_{1}}\right) / t\right)-$ граничное значение непрерывной в $D$ ветви $\arg \left(\left(z-t_{1, k_{1}}\right) / z\right)$, которая при $t=q \exp (i \gamma)$ принимает значение $\theta_{1, k_{1}}=-\pi / 2+$ $\left(\gamma_{1, k_{1}}-\gamma\right) / 2$ при $0 \leqslant \gamma<\gamma_{1, k_{1}}, \theta_{1, k_{1}}=\left(\pi+\gamma_{1, k_{1}}-\gamma\right) / 2$ при $\gamma_{1, k_{1}}<\gamma \leqslant 2 \pi\left(\gamma_{1, k_{1}}=\right.$ $\left.\arg t_{1, k_{1}}\right)$. Тогда с учетом формулы $(2)$ условие (1) можно записать в виде:

$$
\begin{array}{r}
\operatorname{Re}\left(e^{-i\left(\nu(t)+\sum_{j=0,1} \sum_{k_{j}=1}^{p_{j}} m_{j, k_{j}} \theta_{j, k_{j}}(t)\right)} F_{1}(t)\right) \\
=\frac{c(t)|t|^{-\sum_{k_{1}=1}^{p_{1}} m_{1, k_{1}}}}{|G(t)| \prod_{j=0,1} \prod_{k_{j}=1}^{p_{j}}\left|t-t_{j, k_{j}}\right|^{\mu_{j, k_{j}}}},
\end{array}
$$

где $G(t)=a(t)-i b(t), \nu(t)$ - ветвь $\arg G(t)$, выбранная так, чтобы в точках разрыва вьполнялось неравенство $0 \leqslant \nu\left(t_{j, k_{j}}+0\right)-\nu\left(t_{j, k_{j}}-0\right)<2 \pi$ (через $\nu\left(t_{j, k_{j}}-0\right)$, $\nu\left(t_{j, k_{j}}+0\right)$ обозначены пределы, к которым стремится $\nu(t)$, когда точка $t$ стремится к $t_{j, k_{j}}$ соответственно в положительном и отрицательном направлении).

Введем в рассмотрение функцию $\varphi(t)=\nu(t)-\beta(t) \pi$, где $\beta(t)$ целочисленная функция, принимающая значение $\beta_{j, k_{j}}$ на каж дой из дуг $t_{j, k_{j}} t_{j, k_{j}+1}, k_{j}=0, \ldots, p_{j}, j=0,1$. Положив $\beta_{j, 0}=0$, подберем $\beta_{j, k_{j}}$ так, чтобы число $\delta_{j, k_{j}}=\varphi\left(t_{j, k_{j}}+0\right)-\varphi\left(t_{j, k_{j}}-0\right)$ удовлетворяло неравенству $-\pi<\delta_{j, k_{j}} \leqslant 0$, если ищется решение, ограниченное вблизи точки $t_{j, k_{j}}$, и неравенству $0<\delta_{j, k_{j}}<\pi$, если ищется решение, неограниченное вблизи точки $t_{j, k_{j}}$. Полагая $\beta_{j, 0}=0, j=0,1$, получим определенную на всем контуре $L$ функцию $\beta(t)$. При положительном обходе контура $L_{j}$ функция $\varphi(t)$ получит приращение $\varphi\left(t_{j, 0}-0\right)-\varphi\left(t_{j, 0}+0\right)=\aleph_{j} \pi$, где $\aleph_{j}$ - целое число, $j=0,1$.

Рассмотрим функцию $\theta(t)=\sum_{j=0,1} \sum_{k_{j}=1}^{p_{j}} m_{j, k_{j}} \theta_{j, k_{j}}(t)-\beta_{1}(t) \pi$, где $\beta_{1}(t)=0$ на дуге $t_{j, 0} t_{j, 1}, \beta_{1}(t)=-\sum_{k_{j}=1}^{l_{j}} m_{j, k_{j}}$ на дуге $t_{j, l_{j}} t_{j, l_{j}+1}, l_{j}=1, \ldots, p_{j}, j=0,1$. Нетрудно убедиться, что функция $\theta(t)$ непрерывна в точках $t_{j, k_{j}}, k_{j}=1, \ldots, p_{j}, j=0,1$, а при обходе контура $L_{j}$ получит приращение $m_{j} \pi$, причем $m_{j}=\sum_{k_{j}=1}^{p_{j}} m_{j, k_{j}}, j=0,1$.

Число $\aleph_{0}+m_{0}+\aleph_{1}+m_{1}$ будем назьвать индексом задачи, отвечающим данному классу решений. 
1. Пусть $\aleph_{0}+m_{0}$ и $\aleph_{1}+m_{1}-$ четные числа. Введем в рассмотрение функцию

$\psi(t)=\varphi(t)+\theta(t)+\frac{\aleph_{1}+m_{1}}{2} \arg (t)-\left(\frac{\aleph}{2}-1\right) \arg (t-p)-\arg \left(t-z_{1}\right)+\sigma(t) \pi n, \quad t \in L$,

где $z_{1}=r \exp (i \alpha), q<r<1,0 \leqslant \alpha<\pi ; q<p<1 ; \sigma(t)=0$ при $t \in L_{0}$, $\sigma(t)=1$ при $t \in L_{1} ; n$ - целое число; $\arg (t)$ - значение на $L$ ветви $\arg z$, непрерьвной и однозначной в плоскости, разрезанной вдоль линии $y=0, x \geqslant 1 ; \arg (t-p)$ - значение на $L$ ветви $\arg (z-p)$, непрерывной и однозначной в плоскости, разрезанной вдоль линии $y=0, x \geqslant p$, причем $0 \leqslant \arg z<2 \pi, 0 \leqslant \arg (z-p)<2 \pi ; \arg \left(t-z_{1}\right)$ - значение на $L$ определенной ветви $\arg \left(z-z_{1}\right)$, непрерьвной и однозначной в области $D$ с разрезом вдоль линии, соединяющей точки $z=z_{1}$ и $z=1$ и лежащей внутри верхнего полукольца. Здесь $z=p$ - заданная точка, $z=z_{1}$ - точка, положение которой будет определено ниже. Нетрудно убедиться, что функция $\psi(t)$ будет непрерьвной в точках $z=1$ и $z=q$.

С учетом введенных вьше функций условие (3) можно представить в виде

$$
\begin{gathered}
\operatorname{Re}\left(\frac{e^{-i \psi(t)} t^{\frac{\aleph_{1}+m_{1}}{2}} F_{1}(t)}{\left(t-z_{1}\right)(t-p)^{\frac{\aleph}{2}-1}}\right) \\
=\frac{c(t)|t|^{\frac{\aleph_{1}-m_{1}}{2}} \cos \left(\left(\beta(t)+\beta_{1}(t)\right) \pi\right) \cos (\sigma(t) \pi n)}{|G(t)|\left|t-z_{1}\right||t-p|^{\frac{\aleph}{2}-1} \prod_{j=0,1} \prod_{k_{j}=1}^{p_{j}}\left|t-t_{j, k_{j}}\right|^{\mu_{j, k_{j}}}} .
\end{gathered}
$$

Определим однозначную и аналитическую в области $D$ функцию $\Gamma(z)$, граничные значения мнимой части которой равны $\psi(t)$. Такая функция существует, если вьполняется условие однозначности [8, с. 239]

$$
\int_{0}^{2 \pi} \psi(\exp (i \gamma)) d \gamma-\int_{0}^{2 \pi} \psi(q \exp (i \gamma)) d \gamma \equiv \int_{L} \psi(t) \frac{d t}{i t}=0
$$

Условие (5) будет выполняться, если положить

$$
\alpha=-\frac{1}{2 \pi} \int_{L} \varphi(t) \frac{d t}{i t}+\frac{1}{2} \sum_{j=0,1} \sum_{k_{j}=1}^{p_{j}} m_{j, k_{j}} \gamma_{j, k_{j}}+\pi\left(n-m_{0}\right) .
$$

При этом число $n$ следует выбрать так, чтобы $0 \leqslant \alpha<\pi$. Затем с помощью формулы Вилля находим

$$
\Gamma(z)=i S(\psi, z)
$$

где $S(\psi, z) \equiv \frac{\omega}{\pi^{2}} \int_{L} \psi(t)\left[\zeta\left(\frac{\omega}{i \pi} \ln \frac{z}{t}\right)+\sigma(t) \eta^{\prime}\right] \frac{d t}{t}, q=e^{\frac{\pi i \omega^{\prime}}{\omega}}$ (см. [8, с. 238]). Умножая условие $(4)$ на $\exp \left[-\Gamma_{0}(t)\right]$, где $\Gamma_{0}(t)=\operatorname{Re} \Gamma^{+}(t)$, получим

$$
\begin{aligned}
& \operatorname{Re}\left(\frac{e^{-\Gamma^{+}(t)} t^{\frac{\aleph_{1}+m_{1}}{2}} F_{1}(t)}{\left(t-z_{1}\right)(t-p)^{\frac{\aleph}{2}-1}}\right)=c_{1}(t) \\
& \equiv \frac{c(t)|t|^{\frac{\aleph_{1}-m_{1}}{2}} \cos \left[\left(\beta(t)+\beta_{1}(t)\right) \pi\right] \cos (\sigma(t) \pi n) e^{-\Gamma_{0}(t)}}{|G(t)|\left|t-z_{1}\right||t-p|^{\frac{\aleph}{2}-1} \prod_{j=0,1} \prod_{k_{j}=1}^{p_{j}}\left|t-t_{j, k_{j}}\right|^{\mu_{j, k_{j}}}} .
\end{aligned}
$$


Здесь $\Gamma^{+}(t)$ граничное значение функции $\Gamma(z)$ при стремлении $z$ к $t$ из $D$.

a) Пусть $\aleph \geqslant 2$. Используя результаты статьи [9], при $\aleph>2$ получим формулу, дающую решение задачи $(7)$, заменяя в ней функцию $F_{1}(z)$ на выражение, определяемое формулой (2),

$$
\begin{aligned}
F(z)= & \frac{\left(z-z_{1}\right)(z-p)^{\frac{\aleph}{2}} z^{\frac{m_{1}-\aleph_{1}}{2}} e^{\Gamma(z)}}{\prod_{j=0,1} \prod_{k_{j}=1}^{p_{j}}\left(z-t_{j, k_{j}}\right)^{m_{j, k_{j}}}}\left\{S \left(\tilde{c}_{1}, z\right.\right. \\
& \left.+i C_{0}+\sum_{k=1}^{\frac{\aleph}{2}-1} \frac{\mu_{k}^{\prime}+i \mu_{k}^{\prime \prime}}{(z-p)^{k}}+\frac{\nu^{\prime}+i \nu^{\prime \prime}}{z-z_{1}}\right\}
\end{aligned}
$$

где

$$
\begin{aligned}
& \tilde{c}_{1}=\tilde{c}_{1}(t) \equiv c_{1}-\operatorname{Re} \sum_{k=1}^{\frac{\aleph}{2}-1} \frac{\mu_{k}^{\prime}+i \mu_{k}^{\prime \prime}}{(t-p)^{k}}-\operatorname{Re} \frac{\nu^{\prime}+i \nu^{\prime \prime}}{t-z_{1}} \\
& \mu_{1}^{\prime}=\frac{p}{2 \pi} \int_{L} c_{1}(t) \frac{d t}{i t}-\frac{p}{r}\left(\nu^{\prime} \cos \alpha+\nu^{\prime \prime} \sin \alpha\right)-\sum_{k=2}^{\frac{\aleph}{2}-1} \frac{\mu_{k}^{\prime}}{(-p)^{k-1}}
\end{aligned}
$$

$C_{0}, \nu^{\prime}, \nu^{\prime \prime}, \mu_{1}^{\prime \prime}, \mu_{k}^{\prime}, \mu_{k}^{\prime \prime}, k=2, \ldots, \aleph / 2-1,-$ произвольные действительные постоянные. В случае $\aleph=2$ решение будет определятся формулой (8), если в ней положить равными нулю все $\mu_{k}^{\prime}, \mu_{k}^{\prime \prime}$. При этом следует принять

$$
\nu^{\prime}=\frac{r}{2 \pi} \int_{L} \frac{c_{1}(t) d t}{i t}
$$

когда $\alpha=0$, или

$$
\nu^{\prime \prime}=\frac{r}{2 \pi} \int_{L} c_{1}(t) \frac{d t}{i t}-\nu^{\prime} \operatorname{ctg} \alpha,
$$

когда $\alpha>0$.

б) Пусть $\aleph=0$. Условие (3) запишем в виде

$$
\begin{array}{r}
\operatorname{Re}\left(\frac{e^{-i \psi(t)}\left(t-z_{1}\right) t^{\frac{\aleph_{1}+m_{1}}{2}} F_{1}(t)}{(t-p)}\right)=c_{1}(t) \\
\equiv \frac{c(t)\left|t-z_{1}\right||t|^{\frac{\aleph_{1}-m_{1}}{2}} \cos \left(\left(\beta(t)+\beta_{1}(t)\right) \pi\right) \cos (\sigma(t) \pi n)}{|G(t)||t-p| \prod_{j=0,1} \prod_{k_{j}=1}^{p_{j}}\left|t-t_{j, k_{j}}\right|^{\mu_{j, k_{j}}}}
\end{array}
$$

где

$$
\psi(t)=\varphi(t)+\theta(t)+\frac{\aleph_{1}+m_{1}}{2} \arg t-\arg (t-p)+\arg \left(t-z_{1}\right)+\sigma(t) \pi n, \quad t \in L .
$$


Определяя, как и выше, число $\alpha=\arg z_{1}$ так, чтобы функция (10) удовлетворяла условию (5), найдем по формуле (6) функцию $\Gamma(z)$. После этого условие (9) запишем в виде

$$
\begin{aligned}
& \operatorname{Re}\left(\frac{e^{-\Gamma^{+}(t)}\left(t-z_{1}\right) t^{\frac{\aleph_{1}+m_{1}}{2}} F_{1}(t)}{t-p}\right) \\
& =c_{1}(t) \equiv \frac{c(t)\left|t-z_{1}\right||t|^{\frac{\aleph_{1}-m_{1}}{2}} \cos \left(\left(\beta(t)+\beta_{1}(t)\right) \pi\right) \cos (\sigma(t) \pi n)}{|G(t)||t-p| \prod_{j=0,1} \prod_{k_{j}=1}^{p_{j}}\left|t-t_{j, k_{j}}\right|^{\mu_{j, k_{j}}} e^{\Gamma_{0}(t)}} .
\end{aligned}
$$

б1) Пусть $\alpha>0$. Тогда единственное решение задачи определяется следующей формулой

$$
\begin{aligned}
F(z)= & \frac{(z-p) z^{\frac{m_{1}-\aleph_{1}}{2}} e^{\Gamma(z)}}{\left(z-z_{1}\right) \prod_{j=0,1} \prod_{k_{j}=1}^{p_{j}}\left(z-t_{j, k_{j}}\right)^{\mu_{j . k_{j}}}}\left\{S\left(\tilde{c}_{1}, z\right)\right. \\
& \left.+\frac{p}{2 \pi(z-p)} \int_{L} \frac{c_{1}(t) d t}{i t}+i C_{0}+i C_{1} g(z)\right\},
\end{aligned}
$$

где

$$
\tilde{c}_{1}=\tilde{c}_{1}(t) \equiv c_{1}(t)-\frac{p}{2 \pi} \operatorname{Re} \frac{1}{t-p} \int_{L} \frac{c_{1}(t) d t}{i t}
$$

a

$$
g(z)=\frac{\operatorname{sn}^{2}\left(\frac{K \ln p}{\ln q}\right)}{\operatorname{sn}^{2}\left(\frac{K \ln z}{\ln q}\right)-\operatorname{sn}^{2}\left(\frac{K \ln p}{\ln q}\right)}
$$

- функция, конформно отображающая область $D$ на внешность разрезов вдоль двух отрезков вещественной оси. Постоянные $C_{0}$ и $C_{1}$ определяются из условий

$$
\begin{aligned}
& \operatorname{Re} S\left(\tilde{c}_{1}, z_{1}\right)+\frac{p}{2 \pi} \int_{L} \frac{c_{1}(t) d t}{i t} \operatorname{Re} \frac{1}{z_{1}-p}+C_{1} \operatorname{Re}\left(i g\left(z_{1}\right)\right)=0, \\
& \operatorname{Im} S\left(\tilde{c}_{1}, z_{1}\right)+\frac{p}{2 \pi} \int_{L} \frac{c_{1}(t) d t}{i t} \operatorname{Im} \frac{1}{z_{1}-p}+C_{0}+C_{1} \operatorname{Im}\left(i g\left(z_{1}\right)\right)=0 .
\end{aligned}
$$

$\left.\sigma_{2}\right)$ Пусть $\alpha=0$. Положим в условии $(11) z_{1}=p$. Тогда, если выполняется равенство

$$
\int_{L} c_{1}(t) \frac{d t}{i t}=0
$$

решение задачи будет определяться формулой

$$
F(z)=\frac{z^{\frac{m_{1}-\aleph_{1}}{2}} \exp (\Gamma(z))}{\prod_{j=0,1} \prod_{k_{j}=1}^{p_{j}}\left(z-t_{j, k_{j}}\right)^{m_{j, k_{j}}}}\left\{S\left(c_{1}, z\right)+i C_{0}\right\} .
$$

При невыполнении условия (12) задача неразрешима. 
в) Пусть $\aleph \leqslant-2$. При $\aleph<-2$ задача будет разрешима, если выполняются следующие условия

$$
\begin{gathered}
\frac{\omega}{\pi^{2}} \int_{L} c_{1}(t)\left[\zeta\left(\frac{\omega}{\pi^{2}} \ln \frac{p}{t}\right)+\sigma(t) \eta^{\prime}\right] \frac{d t}{i t}+i C_{0}=0 \\
\int_{L} c_{1}(t) \zeta^{(k)}\left(\frac{\omega}{\pi i} \ln \frac{p}{t}\right) \frac{d t}{t}=0, \quad k=1,2 \ldots,-\frac{\aleph}{2}-2, \\
\frac{\omega}{\pi^{2}} \int_{L} c_{1}(t)\left[\zeta\left(\frac{\omega}{\pi i} \ln \frac{z_{1}}{t}\right)+\sigma(t) \eta^{\prime}\right] \frac{d t}{i t}+i C_{0}=0 \\
\int_{L} c_{1}(t) \frac{d t}{t}=0,
\end{gathered}
$$

где

$$
\begin{aligned}
& c_{1}(t)= \frac{c(t)\left|t-z_{1}\right||t|^{\frac{\aleph_{1}-m_{1}}{2}} \cos \left[\left(\beta(t)+\beta_{1}(t)\right) \pi\right] \cos (\sigma(t) \pi n)}{|G(t)||t-p|^{\frac{\aleph}{2}-1} \prod_{j=0,1} \prod_{k_{j}=1}^{p_{j}}\left|t-t_{j, k_{j}}\right|^{\mu_{j, k_{j}}} \exp \left\{\Gamma_{0}(t)\right\}}, \\
& \Gamma_{0}(t)=\operatorname{Re}^{+}(t), \quad \Gamma(z)=i S(\psi, z) \\
& \psi(t)= \varphi(t)+\theta(t)+\frac{\aleph_{1}+m_{1}}{2} \arg t \\
&-\left(\frac{\aleph}{2}+1\right) \arg (t-p)+\arg \left(t-z_{1}\right)+\sigma(t) \pi n .
\end{aligned}
$$

Выделяя в равенствах (13)-(15) действительные и мнимые части, приходим к действительньп соотношениям, одно из которых можно удовлетворить подбором постоянной $C_{0}$. Оставшиеся соотношения и соотношение (16) будут являться условиями разрешимости задачи. В случае выполнения этих условий единственное решение задачи будет определяться формулой

$$
F(z)=\frac{(z-p)^{\frac{\aleph}{2}+1} z^{\frac{m_{1}-\aleph_{1}}{2}} \exp (\Gamma(z))}{\left(z-z_{1}\right) \prod_{j=0,1} \prod_{k_{j}=1}^{p_{j}}\left(z-t_{j, k_{j}}\right)^{m_{j, k_{j}}}}\left\{S\left(c_{1}, z\right)+i C_{0}\right\}
$$

При $\aleph=-2$ решение задачи будет определятся последней формулой, если вьполняется условие (16) и условие, получаемое из (15) выделением действительных частей (выделяя в (15) мнимые части, приходим к соотношению, из которого находится $C_{0}$ ).

2. Пусть $\aleph_{0}+m_{0}-$ нечетное, $\aleph_{1}+m_{1}-$ четное числа. Краевое условие (3) можно записать в виде

$$
\begin{gathered}
\operatorname{Re}\left[\frac{e^{-i \psi(t)} t^{\frac{\aleph_{1}+m_{1}}{2}}(t-1) F_{1}(t)}{(t-p)^{\frac{\aleph-1}{2}}\left(t-z_{1}\right)}\right] \\
=\frac{c(t)|t|^{\frac{\aleph_{1}-m_{1}}{2}}|t-1| \cos \left(\left(\beta(t)+\beta_{1}(t)\right) \pi\right) \cos (\sigma(t) \pi n)}{|G(t)| \prod_{j=0,1} \prod_{k_{j}=1}^{p_{j}}\left|t-t_{j, k_{j}}\right|^{\mu_{j, k_{j}}}\left|t-z_{1}\right||t-p|^{\frac{\aleph-1}{2}}} .
\end{gathered}
$$


Здесь

$$
\begin{aligned}
\psi(t)= & \varphi(t)+\theta(t)+\frac{\aleph_{1}+m_{1}}{2} \arg t-\frac{\aleph-1}{2} \arg (t-p) \\
& +\arg (t-1)-\arg \left(t-z_{1}\right)+\sigma(t) \pi n .
\end{aligned}
$$

В формуле (17) под $\arg (t-1)$ понимается значение на $L$ ветви $\arg (z-1)$, непрерывной и однозначной в плоскости, разрезанной вдоль линии $y=0, x \geqslant 1$, причем $0 \leqslant \arg (z-$ 1) $<2 \pi$.

Определим число $\alpha=\arg z_{1}$ так, чтобы функция (18) удовлетворяла условию (5), и с помощью формулы $(6)$ найдем функцию $\Gamma(z)$. Затем соотношение $(7)$ представим в виде

$$
\begin{aligned}
\operatorname{Re} & {\left[\frac{e^{-\Gamma^{+}(t)}(t-1) t^{\frac{\aleph_{1}+m_{1}}{2}} F_{1}(t)}{(t-p)^{\frac{\aleph-1}{2}}\left(t-z_{1}\right)}\right]=c_{1}(t) } \\
& \equiv \frac{c(t)|t|^{\frac{\aleph_{1}-m_{1}}{2}}|t-1| \cos \left(\left(\beta(t)+\beta_{1}(t)\right) \pi\right) \cos (\sigma(t) \pi n) \exp \left(-\Gamma_{0}(t)\right)}{|G(t)| \prod_{j=0,1} \prod_{k_{j}=1}^{p_{j}}\left|t-t_{j, k_{j}}\right|^{\mu_{j, k_{j}}}\left|t-z_{1}\right||t-p|^{\frac{\aleph-1}{2}}} .
\end{aligned}
$$

а) Пусть $\aleph \geqslant 1$. При $\aleph>1$ по аналогии с пунктом 1 получим

$$
\begin{aligned}
F_{1}(z)= & \frac{\left(z-z_{1}\right)(z-p)^{\frac{\aleph-1}{2}} e^{\Gamma(z)}}{(z-1) z^{\frac{\aleph_{1}+m_{1}}{2}}}\left\{S\left(\tilde{c}_{1}, z\right)+i C_{0}\right. \\
& \left.+\sum_{k=1}^{\frac{\aleph-1}{2}} \frac{\mu_{k}^{\prime}+i \mu_{k}^{\prime \prime}}{(z-p)^{k}}+\frac{\nu^{\prime}+i \nu^{\prime \prime}}{z-z_{1}}\right\},
\end{aligned}
$$

где

$$
\begin{aligned}
& \tilde{c}_{1}=\tilde{c}_{1}(t)=c_{1}(t)-\operatorname{Re} \sum_{k=1}^{\frac{\aleph-1}{2}} \frac{\mu_{k}^{\prime}+i \mu_{k}^{\prime \prime}}{(t-p)^{k}}-\operatorname{Re} \frac{\nu^{\prime}+i \nu^{\prime \prime}}{t-z_{1}}, \\
& \mu_{1}^{\prime}=\frac{p d}{2 \pi}-\frac{p}{r}\left(\nu^{\prime} \cos \alpha+\nu^{\prime \prime} \sin \alpha\right)-\sum_{k=2}^{\frac{\aleph-1}{2}} \frac{\mu_{k}^{\prime}}{(-p)^{k-1}}, \quad d=\int_{L} c_{1}(t) \frac{d t}{i t} .
\end{aligned}
$$

Отсюда и из (19)

$$
\begin{aligned}
F(z)= & \frac{\left(z-z_{1}\right)(z-p)^{\frac{\aleph-1}{2}} z^{\frac{m_{1}-\aleph_{1}}{2}} \exp (\Gamma(z))}{(z-1) \prod_{j=0,1} \prod_{k_{j}=1}^{p_{j}}\left(z-t_{j, k_{j}}\right)^{m_{j, k_{j}}}}\left\{S\left(\tilde{c}_{1}, z\right)\right. \\
& \left.+i C_{0}+\sum_{k=1}^{\frac{\aleph-1}{2}} \frac{\mu_{k}^{\prime}+i \mu_{k}^{\prime \prime}}{(z-p)^{k}}+\frac{\nu^{\prime}+i \nu^{\prime \prime}}{z-z_{1}}\right\} .
\end{aligned}
$$

Для того чтобы функция (20) являлась решением исходной задачи, необходимо соблюдение условия

$$
\frac{\omega}{\pi^{2}} \int_{L} \tilde{c}_{1}(t)\left[\zeta\left(-\frac{\omega \ln t}{\pi i}\right)+\sigma(t) \eta^{\prime}\right] \frac{d t}{i t}+C_{0}+\sum_{k=1}^{\frac{\kappa-1}{2}} \frac{\mu_{k}^{\prime \prime}}{(1-p)^{k}}+\operatorname{Im} \frac{\nu^{\prime}+i \nu^{\prime \prime}}{1-z_{1}}=0 .
$$


Это равенство удовлетворим подбором постоянной $C_{0}$ и из него найдем выражение для $C_{0}$. Тогда решение исходной задачи определяется формулой (20) и будет содержать произвольных постоянных $\nu^{\prime}, \nu^{\prime \prime}, \mu_{1}^{\prime}, \mu_{k}^{\prime}, \mu_{k}^{\prime \prime}, k=2, \ldots,(\aleph-1) / 2$.

При $\aleph=1$ формула (20) будет определять решение исходной задачи, если в ней положить $\mu_{k}^{\prime}=\mu_{k}^{\prime \prime}=0$ для $k \geqslant 1$,

$$
\begin{aligned}
\nu^{\prime \prime} & =\frac{r d}{2 \pi \sin \alpha}-\nu^{\prime} \operatorname{ctg} \alpha \\
C_{0} & =-\frac{\omega}{\pi^{2}} \int_{L} \tilde{c}_{1}(t)\left[\zeta\left(-\frac{\omega \ln t}{\pi i}\right)+\sigma(t) \eta^{\prime}\right] \frac{d t}{i t}-\operatorname{Im} \frac{\nu^{\prime}+i \nu^{\prime \prime}}{1-z_{1}}
\end{aligned}
$$

в случае $\alpha>0$ и $\mu_{k}^{\prime}=\mu_{k}^{\prime \prime}=0$ для $k \geqslant 1, \nu^{\prime}=r d /(2 \pi)$,

$$
C_{0}=-\frac{\omega}{\pi^{2}} \int_{L} \tilde{c}_{1}(t)\left[\zeta\left(-\frac{\omega \ln t}{\pi i}\right)+\sigma(t) \eta^{\prime}\right] \frac{d t}{i t}-\frac{\nu^{\prime \prime}}{1-r}
$$

когда $\alpha=0$. Решение будет содержать произвольную постоянную $\nu^{\prime}$ при $\alpha>0$ или $\nu^{\prime \prime}$ при $\alpha=0$.

б) В случае $\aleph \leqslant-1$ получим

$$
\begin{aligned}
F(z)= & \frac{\left(z-z_{1}\right)(z-p)^{\frac{\aleph-1}{2}} z^{\frac{m_{1}-\aleph_{1}}{2}} \exp (\Gamma(z))}{(z-1) \prod_{j=0,1} \prod_{k_{j}=1}^{p_{j}}\left(z-t_{j, k_{j}}\right)^{m_{j, k_{j}}}}\left\{S\left(\tilde{c}_{1}, z\right)\right. \\
& \left.+i C_{0}+\frac{\nu^{\prime} e^{i \alpha}}{z-z_{1}}+i C_{1} \tilde{g}(z)\right\},
\end{aligned}
$$

где $\tilde{c}_{1}(t)=c_{1}(t)-\operatorname{Re} \nu^{\prime} e^{i \alpha} /\left(t-z_{1}\right), \nu^{\prime}=p d /(2 \pi), \tilde{g}(z)=g\left(z e^{-i \alpha}\right)$. Чтобы формула (21) определяла решение исходной задачи, необходимо соблюдение условия

$$
\frac{\omega}{\pi^{2}} \int_{L} \tilde{c}_{1}(t)\left[\zeta\left(-\frac{\omega \ln t}{\pi i}\right)+\sigma(t) \eta^{\prime}\right] \frac{d t}{i t}+\nu^{\prime} \operatorname{Im} \frac{e^{i \alpha}}{1-z_{1}}+C_{0}+C_{1} \tilde{g}(1)=0
$$

а также условий

$$
\begin{gathered}
\frac{\omega}{\pi^{2}} \int_{L} \tilde{c}_{1}(t)\left[\zeta\left(\frac{\omega \ln p}{\pi i}-\frac{\omega \ln t}{\pi i}\right)+\sigma(t) \eta^{\prime}\right] \frac{d t}{t}+i C_{0}+\frac{\nu^{\prime} e^{i \alpha}}{p-z_{1}}+i C_{1} \tilde{g}(p)=0 \\
\frac{\omega}{\pi^{2}} \int_{L} \tilde{c}_{1}(t) \zeta^{(k)}\left(\frac{\omega \ln p}{\pi i}-\frac{\omega \ln t}{\pi i}\right) \frac{d t}{t}+\frac{\nu^{\prime} e^{i \alpha}(-1)^{k} k !}{\left(p-z_{1}\right)^{k+1}} \\
+i C_{1} \tilde{g}^{(k)}(p)=0, \quad k=1,2 \ldots,-\frac{\aleph-1}{2}-1
\end{gathered}
$$

Условие (22), а также условие, получающееся выделением мнимой части из (23), удовлетворим подбором постоянных $C_{0}$ и $C_{1}$. Условие, получающееся из (23) выделением действительной части, а также условие (24) дают -א действительных условий разрешимости. При их вьполнении решение дается формулой (21).

Оставшиеся два случая, когда $\aleph_{0}+m_{0}$ и $\aleph_{1}+m_{1}$ оба нечетные либо $\aleph_{0}+m_{0}-$ четное, a $\aleph_{1}+m_{1}-$ нечетное числа, рассматриваются аналогично предыдущим. 
3. В качестве примера применения вьшеизложенных результатов рассмотрим задачу о взрыве двух шнуровых зарядов в однородной среде, когда в ней имеется круговой цилиндр, обтекаемьй возникающим при взрыве течением. Будем считать, что заряды в плоскости $\tilde{z}=\tilde{x}+i \tilde{y}$, перпендикулярной их осям, моделируются диполями с моментами $M_{0}$ и $M_{1}$, расположенными на свободной поверхности $\tilde{y}=0$. При этом используется импульсно-гидродинамическая модель [10].

В рамках этой модели жидкость ведет себя как идеальная несжимаемая жидкость во всей занимаемой ею области, а движение будет плоскопараллельным. Свободную поверхность примем за ось $\tilde{x}$, на которой в точках $\tilde{x}_{0}$ и $x_{1}$ расположены заряды, моделируемые диполями. Итак,задан цилиндр радиуса $a_{0}$, его ось расположена на глубине $h\left(h>a_{0}\right)$ от свободной поверхности.

Введем комплексный потенциал $\tilde{w}(\tilde{z})=\tilde{\varphi}(\tilde{x}, \tilde{y})+i \tilde{\psi}(\tilde{x}, \tilde{y})$. Для него имеем следуюшие граничные условия: $\tilde{\varphi}=0$ на оси $о \tilde{x}, \tilde{\psi}=0$ на границе круга. Причем в окрестности точек $\tilde{z}_{0}=\tilde{x}_{0}$ и $\tilde{z}_{1}=\tilde{x}_{1}$ имеем

$$
\tilde{w}\left(\tilde{z}_{1}\right) \approx \frac{i M_{1}}{2 \pi\left(\tilde{z}-\tilde{z}_{1}\right)}, \quad \tilde{w}\left(z_{0}\right) \approx \frac{i M_{0}}{2 \pi\left(\tilde{z}-\tilde{z}_{0}\right)},
$$

где $M_{0}$ и $M_{1}-$ моменты диполей.

Отобразим область течения на кольцо $q<|z|<1$ функцией

$$
z=\frac{\left(\tilde{z}+i \sqrt{h^{2}-a_{0}^{2}}\right)}{\left(\tilde{z}-i \sqrt{h^{2}-a_{0}^{2}}\right)} .
$$

Радиус внутренней окружности $q(q<1)$ будет равен

$$
q=\frac{\left(\sqrt{h+a_{0}}+\sqrt{h-a_{0}}\right)}{\left(\sqrt{h+a_{0}}-\sqrt{h-a_{0}}\right)} .
$$

При этом отображении оси $O \tilde{x}$ соответствует окружность $|z|=1$, точке $x_{0}$ соответствует $t_{0}, \tilde{x}_{1}$ соответствует $t_{1}$ на окружности $|z|=1$. Таким образом, для кольца получили задачу Гильберта $a(t) \tilde{\varphi}(t)-b(t) \tilde{\psi}(t)=c(t)$, причем $a=1, b=0, c=0$ на $|z|=1$ и $a=0, b=-1, c=0$ на $|z|=q$ или

$$
\operatorname{Re}[(a+i b) \tilde{w}]=0
$$

Представим $\tilde{w}(z)$ в виде $\tilde{w}(z)=w_{1}(z) /\left(\left(z-t_{0}\right)\left(z-t_{1}\right)\right)$, где $w_{1}(z)$ - аналитическая и однозначная в кольце $q<|z|<1$ функция. Запишем условие $(25)$ в виде

$$
\operatorname{Re}\left\{e^{-i\left(\nu(t)+\arg \left(t-t_{0}\right)+\arg \left(t-t_{1}\right)\right)} w_{1}(t)\right\}=0,
$$

где $\nu(t)=\arg (a-i b)$.

Введем, как это было сделано ранее, функцию $\varphi_{1}(t)=\nu(t)-\beta(t) \pi$, причем $\beta(t)$ подберем так, чтобы $-\pi<\varphi_{1}\left(t_{j}+0\right)-\varphi_{1}\left(t_{j}-0\right) \leqslant 0(j=0,1)$, так как функция $w_{1}(t)$ ограничена в точках $t_{0}, t_{1}$.

Здесь можно убедиться, что $\aleph_{0}=\aleph_{1}=0, m_{0}=2, m_{1}=0$. Значит, индекс задачи $\aleph=2$, причем $\aleph_{0}+m_{0}=2, \aleph_{1}=0$. Следовательно, можно применить результаты пункта 1. 
Таким образом, решение определяется формулой

$$
\tilde{w}(z)=\frac{\left(z-z_{1}\right) e^{\Gamma(z)}}{\left(z-t_{0}\right)\left(z-t_{1}\right)}\left\{S\left(\tilde{c}_{1}, z\right)+i C_{0}+\frac{\nu^{\prime}+i \nu^{\prime \prime}}{z-z_{1}}\right\}
$$

причем

$$
\tilde{c}_{1}=c_{1}(t)-\operatorname{Re} \frac{\nu^{\prime}+i \nu^{\prime \prime}}{t-z_{1}}=-\operatorname{Re} \frac{\nu^{\prime}+i \nu^{\prime \prime}}{t-z_{1}} .
$$

При этом

$$
\nu^{\prime \prime}=\frac{r}{2 \pi} \int_{L} c_{1}(t) \frac{d t}{i t}-\nu^{\prime} \operatorname{ctg} \alpha
$$

т.е. $\nu^{\prime \prime}=-\nu^{\prime} \operatorname{ctg} \alpha$,

$$
\begin{aligned}
& \Gamma(z)=i S(\psi, z)=i \frac{\omega}{\pi^{2}} \int_{L} \psi(t)\left[\zeta\left(\frac{\omega \ln \frac{z}{t}}{\pi i}\right)+\sigma(t) \eta^{\prime}\right] \frac{d t}{t} \\
& \psi(t)=\varphi(t)+\theta(t)-\arg \left(t-z_{1}\right)+\sigma(t) \pi n
\end{aligned}
$$

Имея комплексный потенциал (27), легко получить в физической плоскости все интересующие гидродинамические характеристики.

\section{СПИСОК ЦИТИРОВАННОЙ ЛИТЕРАТУРЫ}

[1] Комяк И. И., Усс А. Г. Особый случай задачи Гилберта // Изв. АН БССР. Сер. физ. -мат. наук. 1975. №6. С. 27-29.

[2] Гахов Ф. Д. Краевые задачи. М.: Наука, 1979.

[3] Зверович Э. И. Краевые задачи теории аналитических функций в гёльдеровых классах на римановых поверхностях // УМН. 1971. Т. 26. № 1. С. 113-179.

[4] Гуревич М.И. Теория струй идеальной жидкости. М.: Наука, 1979.

[5] Лаврентьев М. А., Шабат Б. В. Проблемы гидродинамики и их математические модели. М.: Наука, 1977.

[6] Мускат М. Течения однородных жидкостей в пористой среде. М.: Мир, 1949.

[7] Ильинский Н. Б., Поташев А. В. Краевые задачи теории взрыва. Казань: Изд-во Казанского университета, 1976.

[8] Ахиезер Н. И. Элементы теории эллиптических функций. М.: Наука, 1970.

[9] Салимов Р. Б. Селезнёв В. В. Решение краевой задачи Гильберта с разрывными коэффициентами для кольца // Труды семинара по краевым задачам. №17. Казань: Изд-во Казанск. ун-та, 1980.

[10] Кузнецов В. М. Математические модели взрывного дела. Новосибирск: Наука, СО АН CCCP, 1977. 